\title{
Special issue on titin and its binding proteins in striated muscle
}

\author{
Amy $\mathrm{Li}^{1,2} \cdot$ Cristobal dos Remedios ${ }^{1}$
}

Received: 14 May 2017 / Accepted: 16 May 2017 / Published online: 5 June 2017

(C) International Union for Pure and Applied Biophysics (IUPAB) and Springer-Verlag Berlin Heidelberg 2017

Titin is the largest known muscle protein with a megaDalton molecular weight and a contour length greater than one micron. One molecule of titin spans half a sarcomere connecting both the M-line and the Z-disc. This giant protein plays a pivotal role in myocardial passive stiffness, structural integrity and stress-initiated signaling pathways. This Special Issue showcases the latest updates on the role of titin and other associated large molecular weight proteins in the health and disease of cardiac muscle.

Reviews for this Special Issue are split into two separate issues to be published in series. Topics for the first issue includes the history of titin, its prevalence in disease, how posttranslational modifications of titin alter the sarcomere passive tension, methods of determining large molecular weight protein isoforms, as well as the pathology and cellular functions of obscurin - a protein that also belongs to this family of giant sarcomeric proteins.

The first article of this special issue by Cris dos Remedios provides a historical viewpoint of the studies that led to the discovery of titin. Titin, originally coined connectin by Koscak Maruyama in 1976, is the third most abundant protein in the sarcomere, after actin and myosin. This review article documents the discovery of titin filaments from the first observations in 1948, through the development of the sliding filament hypothesis, to theories involving hyperstretched fi-

Amy Li

amy.li@sydney.edu.au

1 Discipline of Anatomy \& Histology, School of Medical Sciences, University of Sydney, Sydney 2006, Australia

2 Department of Molecular Physiology \& Biophysics, University of Vermont, Burlington, VT 05405, USA bers, a range of selective extraction procedures on myofibrils and myofilaments, and serial sections through a single sarcomere, to finally arrive at today's understanding that titin filaments are an integral component of the sarcomere.

Titin mutations are now strongly implicated in cardiomyopathy. Tabish et al. review the association of truncating mutations in the titin gene (TTN) and its implications in dilated cardiomyopathy (DCM). The human TTN gene consists of 363 exons that encode over 38,000 amino acid residues. TTN truncating mutations are found in over a quarter of the familial DCM patient cohort, but perhaps what is more surprising is that $2-3 \%$ of the general "healthy" population also carries these mutations. Tabish et al. suggest that the location of mutation alters the gene-environment interactions as well as other factors that may contribute to disease pathogenicity and severity.

The review by Hamdani and colleagues, on titin posttranslational modification, focuses on titin phosphorylation and titin-based passive tension in samples from patients with heart failure, as well as animal models of heart disease. The authors also discuss the effects of site-specific phosphorylation in modulating stretch-induced spring force of titin, and titin-based passive tension development in cardiomyocytes.

Vikhlyantsev summarizes the nuances and technical considerations of the low-density SDS-PAGE gels used to identify titin and its isoforms. This review centers on techniques that preserve the primary structure of titin to allow for the visualization of its high molecular weight isoforms.

Kellermayer et al. presents an overview of the three novex isoforms found in the human titin gene. They focus on novex3 that encodes the "tiny titin" isoform. In the heart, novex-3 forms a complex with obscurin at the Z-disc and may regulate a number of myofibrillar calcium and signaling pathways. The authors propose that novex-3 may be involved in stressinitiated remodeling of the sarcomere. 
Marston switches gears to discuss obscurin, another member of the giant protein family implicated in inherited cardiomyopathies. More and more mutations have been identified in cardiomyopathies as exome and gene sequencing becomes more affordable. The increasing hit rate of mutations in the obscurin gene $(O B S C N)$ in heart disease has incentivized a number of groups to study this area.

To conclude the first half of this special issue, Manring et al. reviews the role of the multiple obscurin protein iso- forms. The authors discuss the function of obscurin isoform in relation to their expression profile and subcellular distribution in striated muscle.

Acknowledgements We would like to extend our sincerest appreciation to all the authors for their efforts and promptness in writing and amending of their contributions. We also thank the external reviewers for their input and expertise, and the BREV Editorial Office for their assistance in producing this special issue. 